\title{
Two phase flow bifurcation due to turbulence: transition from slugs to bubbles
}

\author{
Grzegorz Górski ${ }^{1}$, Grzegorz Litak ${ }^{2,3, a}$, Romuald Mosdorf ${ }^{1}$, and Andrzej Rysak ${ }^{2}$ \\ 1 Faculty of Mechanical Engineering, Bialystok University of Technology, Wiejska 45C, 15-351 Bialystok, Poland \\ 2 Faculty of Mechanical Engineering, Technical University of Lublin Nadbystrzycka 36, 20-618 Lublin, Poland \\ ${ }^{3}$ Laboratoire de Génie Electrique et Ferroélectricité, Institut National des Sciences Appliquées Lyon, 8 rue de la Physique, \\ 69621 Villeurbanne cedex, France
}

Received 27 March 2015 / Received in final form 21 June 2015

Published online 23 September 2015

(C) The Author(s) 2015. This article is published with open access at Springerlink.com

\begin{abstract}
The bifurcation of slugs to bubbles within two-phase flow patterns in a minichannel is analyzed. The two-phase flow (water-air) occurring in a circular horizontal minichannel with a diameter of $1 \mathrm{~mm}$ is examined. The sequences of light transmission time series recorded by laser-phototransistor sensor is analyzed using recurrence plots and recurrence quantification analysis. Recurrence parameters allow the two-phase flow patterns to be found. On changing the water flow rate we identified partitioning of slugs or aggregation of bubbles.
\end{abstract}

\section{Introduction}

The identification of flow patterns in minichannels often depends on the subjective evaluation of the observer and the experimental technique [1-3] employed. To increase the ability to identification and interpretation, Wang et al. [4], used the non-linear methods including the Hurst and Lyapunov exponents, correlation dimension, and pseudo-phase-plane trajectory to analyze the pressure fluctuations of two-phase flow through a T-junction. It was possible to classify the characteristic flow structures of air in water. Jin et al. [5] showed that the correlation dimension and Kolmogorov entropy were suitable to identify the flow patterns of an oil/water mixture. Nonlinear approaches in temperature and pressure fluctuations of boiling water in minichannels flow were applied by Mosdorf et al. [6], who estimated the correlation coefficient, correlation dimension and largest Lyapunov exponent and reconstructed the attractor for unstable boiling in parallel silicon microchannels. Wang et al. [7] used nonlinear based statistics, to identify the main flow patterns of an oil-gas-water mixture. In particular, the combinations of Lempel-Ziv complexity and approximate entropy have been used for pattern classification.

The methods of Recurrence Plots (RP) and Recurrence Quantification Analysis (RQA) have been of interest to characterize the non-linear dynamics of the flow of oilwater mixtures [8] and is an efficient approach to classify

\footnotetext{
${ }^{a}$ e-mail: g.litak@pollub.pl
}

of flow complexity. Following these studies Faszczewski et al. [9] used the RP and RQA to analyze the flow patterns in a vertical minichannel. The subject of their studies was the application of multi-criterion methods to identify the types of flow patterns which occur during the movement of the liquid-gas mixture in the minichannel. In the current paper we extend this analysis focusing on flow bifurcation and slug (in the form of an fairly elongated bubble) to bubbles transition.

\section{Experimental setup and measurement results}

We have undertaken the experiments by decreasing quasistatically the water volume flow rate $q$ with a constant air volume flow rate $q_{a}=0.0216 \mathrm{l} / \mathrm{min}$ through a horizontal cylindrical transparent minichannel with a diameter of $1 \mathrm{~mm}$. The flow was recorded by a digital camera and additionally laser transmission light was measured with a sampling frequency of $5 \mathrm{kHz}$.

The scheme of the experimental approach is shown in Figure 1. The compressed air generated by a pump (6), passes through the tank (14), valve (10), constant pressure air tank (9) rotameter (7) and a micro bubble generator (8). The scheme of the laser-phototransistor sensor is shown in Figure $1 b$.

The camera snapshots for increasing $q$ are presented in Figure 2. Slugs are represented as elongated cylindrical 
(a)

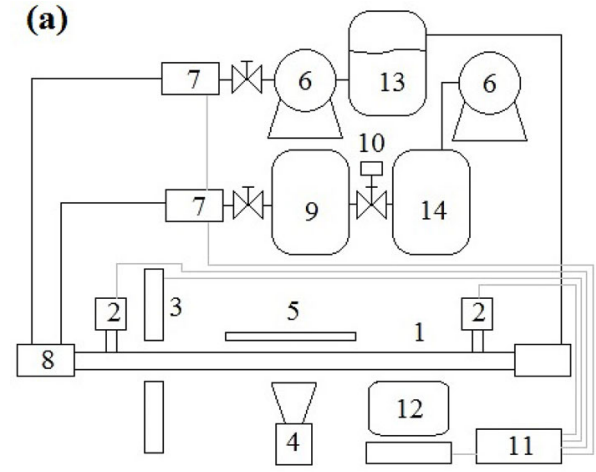

(b)

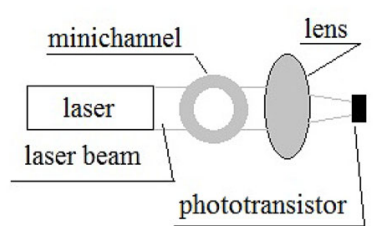

Fig. 1. Schematic of experimental stand. (a) Experimental setup: 1. horizontal minichannel with a diameter of $1 \mathrm{~mm}, 2$. pressure sensors (MPX12DP), 3. laser-phototransistor sensor, 4. Phantom v. 1610 digital camera, 5. lighting, 6. pumps (air or water), 7. flow meters, 8. mini bubble generator, 9. air tank, 10 . automatic valve to maintain a constant pressure in the tank 9 , 11. data acquisition station (DT9800), 12. computer, 13. water tank, 14 air tank. (b) Schematic of laser-phototransistor sensor.

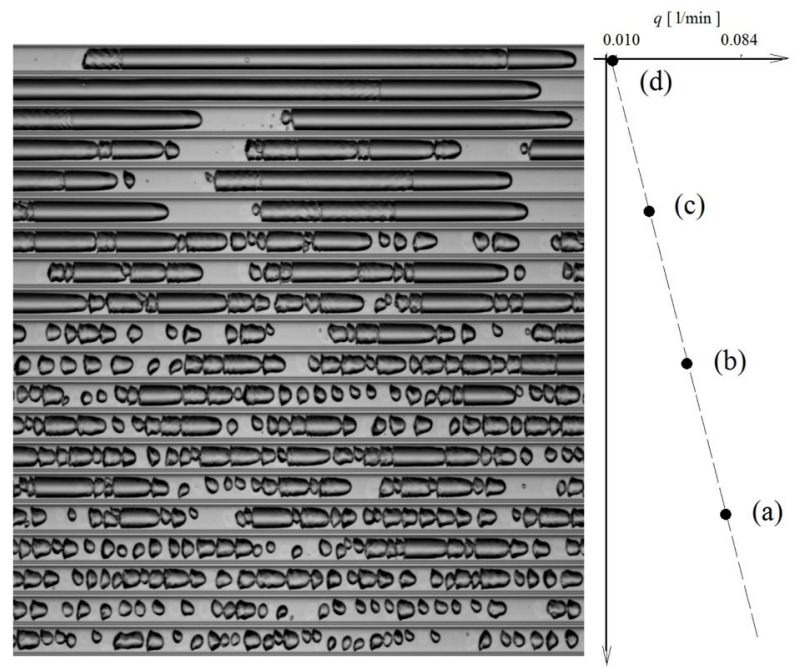

Fig. 2. Sequence of the air-flow flashes for different water volume flow rates $q$ (increasing downward), (a) - (d) and $q=0.068$ (a), 0.049 (b), 0.029 (c), 0.010 l/min (d), respectively.

air structures while bubbles are smaller and more spherical ones. Two interesting features can be found: (a) reduction of the slugs with increasing $q$, (b) a change in the regularity (periodicality) in the mass (air-water) transport. To quantify the flows we analyze the corresponding time series of laser light transmission. The selected cases are presented in Figure 3. Note that light transmission are effected by the content of air in a number of ways. Firstly, we can notice that water volume has a better transmis-
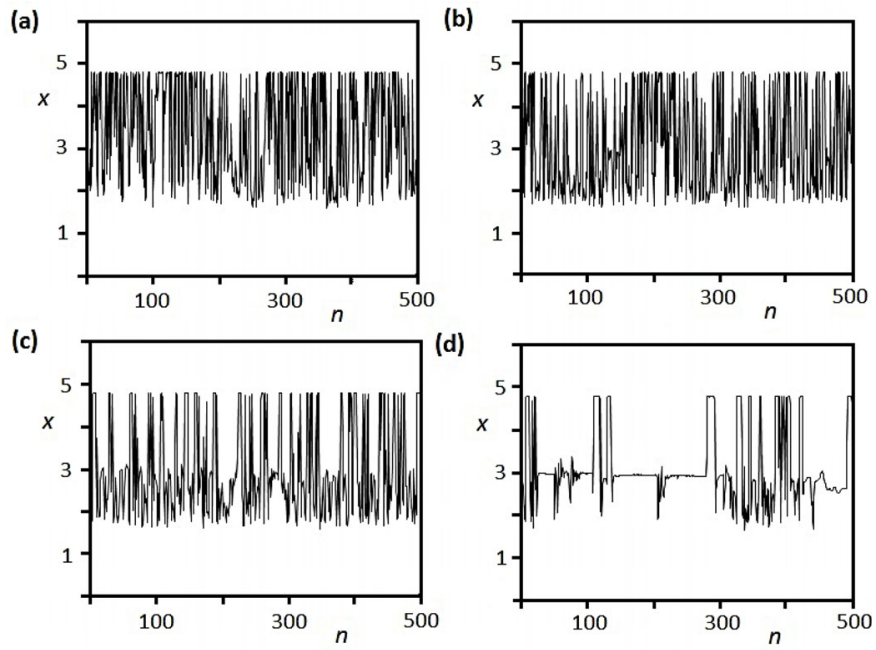

Fig. 3. Laser transmission time series $x[\mathrm{~V}]$ for four chosen cases from (Fig. 2). Water flow rates are $q=0.068$ (a), 0.049 (b), 0.029 (c), $0.010 \mathrm{l} / \mathrm{min}(\mathrm{d})$, with a constant air volume flow rate $q_{a}=0.0216 \mathrm{l} / \mathrm{min}$. Notations $(\mathrm{a})-(\mathrm{d})$ correspond to the cases denoted in Figure 2.

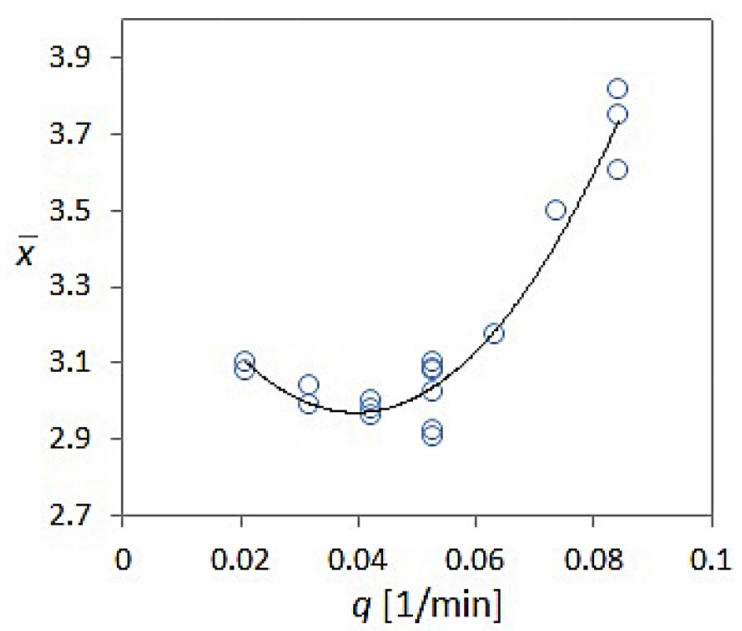

Fig. 4. Average laser transmission $\bar{x}[\mathrm{~V}]$ versus water volume flow rate $q$.

sion than the air volume (Figs. 3a-3d evolve from waterrich to water-poor conditions). However, the above mentioned effect is changed by the onset of fluctuating shapes in the slugs and bubbles as well as their edges during passing through the minichannel (note, the high level of Figs. 3a-3c).

It should be noted that the complexity in the air bubbles and slugs formation contributes to increasing levels of light scattering (Fig. 3) and therefore a decrease in laser light transmission. Consequently, the above feature is also reflected in Figure 4. Here we show the average light transmission $\bar{x}$ versus water volume flow rate $q$. Interestingly, this curve is not a monotonic function. Partially, this is an effect of defragmentation of slugs but also the change in the flow regularity. We study these phenomena more systematically in the next section. 


\section{Recurrence plot and recurrence quantification analysis}

The periodicity of the underlying dynamics can be investigated by means of recurrences which are calculated for each point of the reconstructed trajectory. This method was developed by Eckmann et al. [10] and extended by Webber and Zbilut [11], Casdagli [12] later by Marwan et al. [13-15] and others. This method can be applied for both short deterministic and noise experimental data (see the data analysis from the technical systems [16,17]). Two points on a trajectory are marked as neighbours if they are close enough to each other and this can be expressed by the recurrence matrix $\mathbf{R}^{\epsilon}$ with its element $R_{i j}^{\epsilon}$ given by reference [10]:

$$
R_{i j}^{\epsilon}=\Theta\left(\epsilon-\left\|\mathbf{x}_{i}-\mathbf{x}_{j}\right\|\right),
$$

where $\epsilon$ is the threshold value and $\Theta($.$) denotes the$ Heaviside function. The number of recurrence points depends on both the underlying dynamics and the threshold value $\epsilon$. A standard technique for approximations is that the threshold value should not give more recurrences than a few percentage of the total number of points [14].

Following Takens [18] we define the following vectors $\mathbf{x}$ based on the laser light transmission $x(t)$ which can be written in an embedded space:

$$
\begin{aligned}
\mathbf{x}(t)=(x(t), x(t-\delta i \Delta t), x( & t-2 \delta i \Delta t), \\
& \ldots, x(t-(m-1) \delta i \Delta t),
\end{aligned}
$$

where $\Delta t=0.2 \mathrm{~ms}$ is a sampling period.

The embedding dimension $m=8$, and the delay in the reconstructed coordinates $\delta i=8$ is estimated by the standard methods using the first minimum of averaged mutual information and nodal fraction of the false neighbours [19-21].

Figure 5 compares recurrence plots (RP) for given $\epsilon$, $\epsilon=3 \sigma$, calculated with the Euclidean distance in a frame of the reconstructed phase space. The horizontal and vertical axes represent the time instants $i$ and $j$ for which the distance formula (Eq. (4)) is applied. The presented cases correspond to those denoted by (a) $-(d)$ in Figures 2 and 3 . Therefore Figure $5 \mathrm{a}$ is characterized by a large number of short slugs rarely mediated by bubbles. The formations of black squares (like a checkerboard) could correspond to intermittences $[22,23]$ corresponding to the self organization phenomenon. In Figures $5 \mathrm{~b}$ and $5 \mathrm{c}$ the black squares disappear and correspond to the larger complexity in bubble and slug sizes and their speeds. The intermittency returns in Figure 5d and the slugs are relatively long. Consequently, the RP structure exhibits more clear checkerboard structures. Namely, long slug formation are separated by water content and in the laser transmission one can have the intermittent switching between two states. However the nature of this switching seems to differ from the periodic small slugs recorded in Figures 2d, 3d, and $5 \mathrm{~d}$.
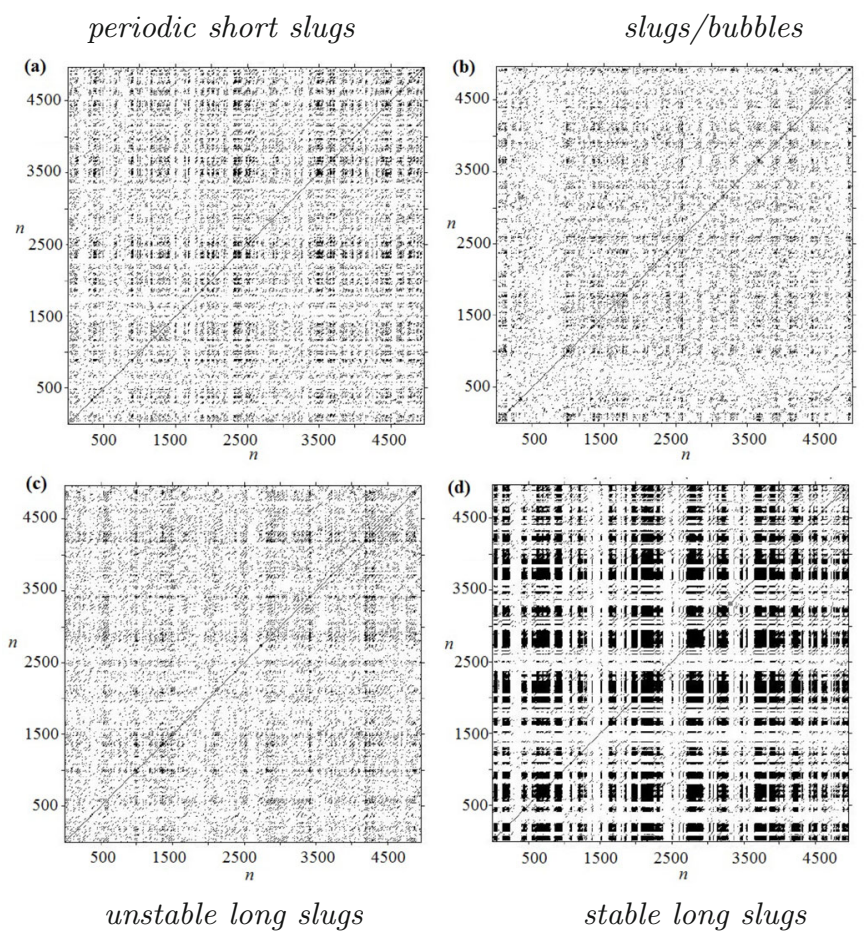

Fig. 5. RP for laser transmission for the cases (a)-(d) specified in Figures 2 and 3. The dimension of the embedding space was $m=9$, while the delay in the reconstructed coordinates $\delta i=5 \Delta t$, where $\Delta t=0.2 \mathrm{~ms}$ is the sampling time, respectively. The threshold was $\epsilon=3 \sigma$.

An additional approach can be employed to quantify and compare the dynamics of a system with a systematic change of $q$. Webber and Zbilut [11] and later Marwan et al. [13,14] developed the Recurrence Quantification Analysis (RQA) for recurrence plots. The RQA analysis includes the recurrence rate variable, $R R$, which is a measure of ability for the system to return to the neighbourhood of a previous state and has the following definition,

$$
R R=\frac{1}{N^{2}} \sum_{i, j \neq i}^{N} R_{i j}^{\epsilon} .
$$

Furthermore, the RQA can be used to identify diagonal and vertical lines through their maximal lengths $L_{\max }$, $V_{\max }$ for diagonal and vertical lines, respectively. The RQA provides the probability $p(l)$ or $p(v)$ of a line distribution according to their lengths $l$ or $v$ (for diagonal and vertical lines). They are calculated as:

$$
p(z)=\frac{P^{\epsilon}(z)}{\sum_{x=x_{\min }}^{N} P^{\epsilon}(z)},
$$

where $z=l$ or $v$ depending on diagonal or vertical structures in the specific recurrence plot. $P^{\epsilon}(z)$ denotes the histogram of $z$ lengths and a fixed value of $\epsilon$.

RQA measures such as: determinism $D E T$, laminarity $L A M$, trapping time $T T$, average length $L$, are based 
on probabilities $P^{\epsilon}(z)$ :

$$
\begin{aligned}
D E T & =\frac{\sum_{l=l_{\min }}^{N} l P^{\epsilon}(l)}{\sum_{l=1}^{N} l P^{\epsilon}(l)}, \\
L A M & =\frac{\sum_{v=v_{\min }}^{N} v P^{\epsilon}(v)}{\sum_{v=1}^{N} v P^{\epsilon}(v)}, \\
L & =\frac{\sum_{l=l_{\min }}^{N} l P^{\epsilon}(l)}{\sum_{l=l_{\min }}^{N} P^{\epsilon}(l)}, \\
T T & =\frac{\sum_{v=v_{\min }}^{N} v P^{\epsilon}(v)}{\sum_{v=v_{\min }}^{N} P^{\epsilon}(v)}, \\
\operatorname{ENTR}_{L} & =-\sum_{l=l_{\min }}^{N} p(l) \log p(l),
\end{aligned}
$$

where $l_{\min }$ and $v_{\min }\left(\right.$ here $\left.l_{\min }=v_{\min }=2\right)$ denote minimal values which should be chosen for a specific dynamical system. The determinism DET is a measure of the predictability of the examined dynamical system and provides the ratio of the recurrence points formed in diagonals to all recurrent points. Note that in a periodic system all recurrence points would be included in the lines parallel to the main diagonal. On the other hand, the laminarity $L A M$ is a similar measure which corresponds to points formed in vertical lines. This measure indicates the dynamics behind sampling point changes. For small (slow) point-to-point changes the consecutive points form a vertical line. Finally, the average diagonal length $L$ refers to the predictability time of a dynamic system while the trapping time $T T$ indicates the average length of the vertical lines measuring the time scale (in terms of sampling intervals) of small changes in the examined time history. Furthermore, the recurrence of the first kind [24,25], $T^{1}$ is an average of empty space between the points. This measure is related the mean period of the dynamical system. In analogy to the probabilities of the diagonal and vertical lengths distributions it is possible to estimate the probability distributions of periods $t$ between the diagonal lines $p_{t}(t)$ and estimate the recurrence period density entropy

$$
E N T R_{t}=-\sum_{l=l_{\min }}^{N} p_{t}(t) \log p_{t}(t) .
$$

$T^{1}$ together with $E N T R_{t}$ are very sensitive to periodic responses of the system and can detect some bifurcations as period doubling and intermittency.

RNA (Recurrence Network Analysis) measures such as: clustering coefficient $C$ and transitivity $T R A N$ are based on the recurrence matrix elements $\mathbf{R}^{\epsilon}[26]$ :

$$
\begin{aligned}
C & =\frac{1}{N} \sum_{i=1}^{N} \frac{\sum_{j, k=1}^{N} A_{j k}^{\epsilon} A_{i j}^{\epsilon} A_{i k}^{\epsilon}}{\sum_{j, k=1}^{N} A_{i j}^{\epsilon} A_{i k}^{\epsilon}\left(1-\delta_{j k}\right)}, \\
T R A N & =\frac{\sum_{i, j, k=1}^{N} A_{j k}^{\epsilon} A_{i j}^{\epsilon} A_{i k}^{\epsilon}}{\sum_{i, j, k=1}^{N} A_{i j}^{\epsilon} A_{i k}^{\epsilon}\left(1-\delta_{j k}\right)},
\end{aligned}
$$

where

$$
A_{i j}^{\epsilon}=R_{i j}^{\epsilon}-\delta_{i j}
$$

The clustering coefficient, $C$, measures the mean fraction of triangles that include the different vertices of the recurrence network, while $T R A N$ is related to the network complexity and can distinguish regular from irregular dynamics.

Note that by introducing the above statistical definitions it is possible to parametrize any RP of the physical data. We have done such a parametrization along the change of the water volume rate flow $q$. The results are presented in Figure 6.

The first observation from Figure 6 is related to the limit of small water volume rate of flow $q$. In this case we see larger values of recurrence rate by 5 times, laminarity by 5 times, determinism by 7 times and also the trapping time by 8 times, comparing to the rest values, which are signaling that the system behaves fairly regular. The flow, in this limit, is related to very long slugs (Fig. 2) and in the laser light transmission we observe a collection of long (Fig. 3d) quasistatic intervals which generate very stable dynamics. This is the most important feature for this limit.

Interestingly, the chosen RQA coefficients show highly non-monotonic behaviour (Fig. 6). This is particularly clear from the divergence $\left(1 / L_{\max }-\right.$ Fig. $\left.6 \mathrm{f}\right), T^{1}$ (recurrence of the first type - Fig. 6i), and from ENT $R_{t}$ (recurrence period density entropy- Fig. 6j) where characteristic two large peaks are formed. By reaching higher values, these measures indicate the non-regular (chaotic) character of the two-phase flow. In the same time, the characteristic minimum between the maxima is associated with the slightly higher values of $R R, D E T, E N T R_{L}, L A M$, $C, T R A N$ (see Figs. 6a, 6b, 6d, 6e, 6k, and 6l, respectively). That is the information that the stochasticity of the system decreases. Namely, the system becomes more deterministic/regular here. To estimate the errors of the above RQA calculations we used the method proposed by Schinkel et al. [27]. It appeared that in the examined cases the error is not significant. It is three orders of magnitude smaller than the RQA parameter values.

\section{Conclusions}

Evidently, in our case, a small divergence $\left(1 / L_{\max }\right)$ is related with large determinism $D E T$ and vice versa (see Fig. 7). Note that these two parameters are sensitive for regular-chaotic and deterministic-stochastic properties [14,28]. Most cases have low determinism and low $1 / L_{\max }$ which implies that the dynamical system is mainly governed by stochastic process. As it clear from Figure 6 the level of stochasticity is not the same showing the small window with series of small regular slugs flow for $q \approx 0.065$ (Fig. 2a).

The results can be concluded as follows. After fairly stable flow in the limit of small $q \rightarrow 0$ with a fairly long air slugs flow the systems is defragmented for larger $q$. On increasing $q$ the system becomes less stable since the slugs 

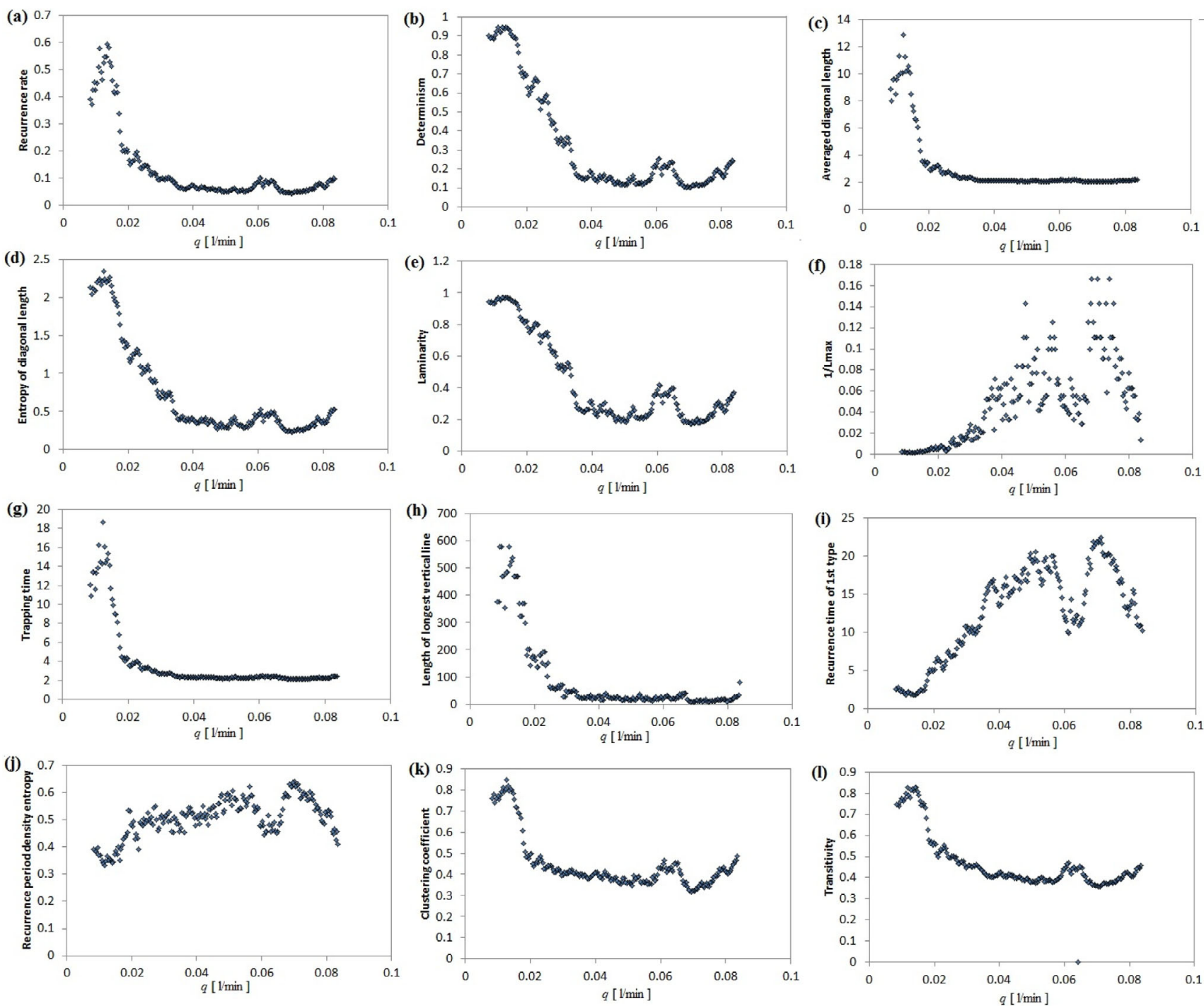

Fig. 6. RQA parameters versus the water volume rate flow, $q$, for laser transmission: recurrence rate $-R R$ (a), determinism $D E T$ (b), average diagonal length $-L(\mathrm{c})$, entropy of diagonal lengths $-E N T R_{L}$ (d), laminarity $-L A M$ (e), divergence $1 / L_{\max }(\mathrm{f})$, trapping time $-T T(\mathrm{~g})$, length of the longest vertical line $-V_{\max }(\mathrm{h})$, recurrence of the first type $-T^{1}$ (i), recurrence period density entropy $-E N T R_{t}(\mathrm{j})$, clustering coefficient $-C(\mathrm{k})$, transitivity $-T R A N(\mathrm{l})$. The calculations of RQA parameters were done by using the Matlab toolbox developed by Marwan [29]. The number of the sampled points was 5000 (as in Fig. 5).

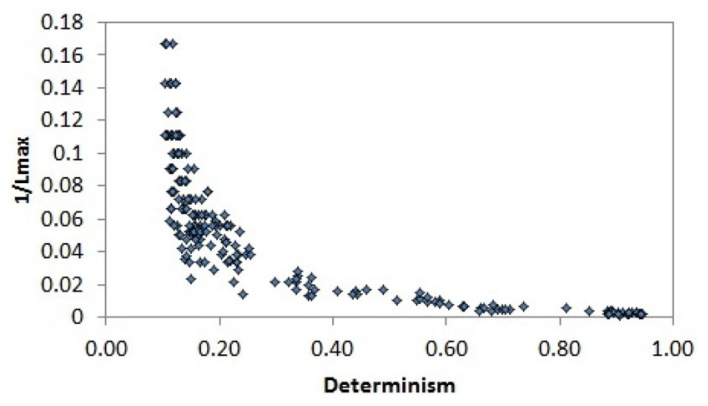

Fig. 7. Divergence $-1 / L_{\max }$ versus determinism $-D E T$.

break into smaller pieces. Rather then long slugs we observe air slugs and air bubbles of different sizes. Obviously, they are moving in the water environment with different speeds. For the condition $q \approx 0.065$ (Fig. 5a) we observe the spectacular effect of structure self-organizing which corresponds to formation of series of short slugs with repeatable sizes (see Fig. 2). Interestingly, the parameters: determinism - DET and laminarity - LAM are very low but they are slightly increased around $q \approx 0.065$. The above tendency is confirmed also by the topological structure of the RPs measured by clustering (Fig. 6k) transitivity - TRAN (Fig. 61).

This small increase could be a spacio-temporal effect similar to a coherent resonance in a noisy system [30]. Here the turbulent based defragmentation of slugs generate an increasingly stochastic component while the geometry of minichannel with the determined diameter allows the resonating air formations of the specific length and volume. 
This research was funded by the National Science Centre, Poland - the number of decision: DEC-2013/09/B/ST8/ 02850 .

\section{References}

1. L. Zhao, K.S. Rezkallah, Int. J. Multiphase Flow 19, 751 (1993)

2. S. Wongwises, M. Pipathattakul, Exp. Thermal Fluid Sci., 30, 345 (2006)

3. L. Chen, Y.S. Tian, T.G. Karayiannis, Int. J. Heat Mass Transfer 49, 4220 (2006)

4. S.F. Wang, R. Mosdorf, M. Shoji, Int. J. Heat Mass Transfer 46, 1519 (2003)

5. N.D. Jin, X.B. Nie, Y.Y. Ren, X.B. Liu, Flow Meas. Instrum. 14, 169 (2003)

6. R. Mosdorf, P. Cheng, H.Y. Wu, M. Shoji, Int. J. Heat Mass Transfer 48, 4667 (2005)

7. Z.Y. Wang, N.D. Jin, Z.K. Gao, Y.B. Zong, T. Wang. Chem. Eng. Sci. 65, 5226 (2010)

8. Y.-B. Zong, N.-D. Jin, Z.-Y. Wang, Z.-K. Gao, C. Wang. Int. J. Multiphase Flow 36, 166 (2010)

9. M. Faszczewski, G. Gorski, R. Mosdorf, Acta Mechanica et Automatica 6, 31 (2012)

10. J.-P. Eckmann, S.O. Kamphorst, D. Ruelle, Europhys. Lett. 5, 973 (1987)

11. C.L. Webber, Jr., J.P. Zbilut, J. Appl. Physiol. 76, 965 (1994)

12. M. Casdagli, J. R. Stat. Soc. 54, 303 (1992)

13. N. Marwan, Encounters with Neighbours: Current Development of Concepts Based on Recurrence Plots and their Applications, Ph.D. Thesis, Universitaet Potsdam, 2003

14. N. Marwan, M.C. Romano, M. Thiel, J. Kurths, Phys. Rep. 438, 237 (2007)

15. N. Marwan, J.F. Donges, Y. Zou, R V. Donner, J. Kurths, Phys. Lett. A 373, 4246 (2009)
16. G. Litak, A. Syta, J. Gajewski, J. Jonak, Meccanica 45, $603(2010)$

17. L.P. Yang, S.L. Ding, G. Litak, E.Z. Song, X.Z. Ma, Chaos 25, 013105 (2015)

18. F. Takens, Detecting Strange Attractors in Turbulence, in Dynamical Systems and Turbulence, Lecture Notes in Mathematics (Springer-Verlag, 1981)

19. A.M. Fraser, H.L. Swinney, Phys. Rev. A 33, 1134 (1986)

20. M.B. Kennel, R. Brown, H.D.I. Abarbanel, Phys. Rev. A 45, 3403 (1992)

21. H. Kantz, T. Schreiber, Non-linear Time Series Analysis (Cambridge University Press, Cambridge, 1997)

22. K. Klimazewska, J.J. Zebrowski, Phys. Rev. E 80, 026214 (2009)

23. G. Litak, A. Syta, B.F. Yao, G.X. Li, J. Theor. Appl. Mech. 47, 55 (2009)

24. J.B. Gao, Phys. Rev. Lett. 83, 3178 (1999)

25. M.S. Baptista, E.J. Ngamga, P.R.F. Pinto, M. Brito, J. Kurths, Phys. Lett. A 374, 1135 (2010)

26. R.V. Donner, J. Heitzig, J.F. Donges, Y. Zou, N. Marwan, J. Kurths, Eur. Phys. J. B 84, 653 (2011)

27. S. Schinkel, N. Marwan, O. Dimigen, J. Kurths, Phys. Lett. A 373, 2245 (2009)

28. N. Marwan, J. Kurths, S. Foerster, Phys. Lett. A 379, 894 (2015)

29. N. Marwan, Cross Recurrence Plot Toolbox for Matlab, Ver. 5.15, Release 28.10, http://tocsy.pik-potsdam.de

30. A.S. Pikovsky, J. Kurths, Phys. Rev. Lett. 78, 775 (1997)

Open Access This is an open access article distributed under the terms of the Creative Commons Attribution License (http://creativecommons.org/licenses/by/4.0), which permits unrestricted use, distribution, and reproduction in any medium, provided the original work is properly cited. 